\title{
Network load traffic on MySQL atomic transaction database
}

\author{
Asghaiyer Mohamed Asghaiyer* \\ Beni Ulid Technology, Bani Walid, Libya \\ asghier86@gmail.com* \\ *corresponding author
}

ARTICLE INFO ABSTRACT

Article history

Received January 5, 2020

Revised January 25, 2020

Accepted February 2, 2020

Keywords

Network load traffic

Mysq

Atomic transaction.
Internet technology is developing very rapidly especially on the database system. Today's database has led to data that cannot be processed into the traditional way that we call big data. Some data stored on the server requires a way for the data to be valid and intact for that transaction mechanism appears on RDBMS which ensures that the data stored will become a unified whole as in customer account data, withdrawal of money at ATMs, e-transactions-commerce and so on. Of course the use of transactions in a database by not using Atomic transactions has a difference in terms of traffic on the network. This research appears by analyzing network traffic or density from a database that uses transactions and not to users who access them. This research method uses a questionnaire method by distributing questionnaires quantitatively to 300 respondents. The results of the study of approximately 300 respondents, researchers get the results that the use of transactions in databases and databases without transactions after being accessed by 300 people, the densest network is a network owned by a system that uses transaction features, this is because there is a slight increase of about $13 \%$ of traffic when compared to a network without transactions. This statement shows that two-way communication from a database that has the transaction provides feedback to the user so that the data is reliable as an indicator that the data has been stored safely. Further research can be done by finding other information or a study of big data using the atomic transaction model.

\section{Introduction}

Network Traffic is the amount of data that moves on the network at a certain point in time [1]. The quality of service in the network is determined by the appropriate network traffic organization. Network data is mostly encapsulated in network packets, which provide a load in the network. Data in the present period is growing very quick, both in terms as volume, velocity and variety. As well as techniques or ways to organize it.

The database has now created information that can't be handled into the customary way we call big data. Big Data is the development or activity of associations to recover, store, process, and examine information that was beforehand impractical or prudent to recover, store, process, and investigate [2], [3]. Big data is data with a large volume so it can't be handled utilizing customary techniques, both organized data and unstructured data. So it needs special techniques to deal with the data.

With the volume and velocity of data, it becomes difficult to keep the data to be valid and intact while transferring data. By using transactions in MySQL can ensure consistency of the data being stored. Use of transactions in a database by not using Atomic transactions has a difference in terms of traffic on the network. For this research appears by analyzing network traffic or density from a database that uses transactions. 
With the background mentioned above this research finally emerged. This research examines the comparison of network load in databases using transactions and databases without transaction.

\section{The Proposed Method/Algorithm}

\subsection{Network Traffic}

According to Technopedia, a trusted website for professional IT insight and inspiration, Network Traffic also known as data traffic alludes to the measure of information moving over a system at a given purpose of time [4]. Network data is for the most part epitomized in network packets, which give the heap in the network. Network traffic is the principle segment for network traffic measurement, network traffic control and recreation [1]. The best possible association of system traffic helps in guaranteeing the nature of administration in a given network [4]. In this topic, an analysis of network traffic can provide benefits to an organization such as:

- Recognizing system bottlenecks - There could be clients or applications that expend high measures of transmission capacity, in this manner comprising a significant piece of the system traffic. Various arrangements can be actualized to handle these.

- System security - Unusual measure of traffic in a system is a conceivable indication of an assault. System traffic reports give significant bits of knowledge into avoiding such assaults.

- System building - Knowing the utilization levels of the system enables future prerequisites to be broke down.

\subsection{MySQL}

SQL or stands for Structured Query Language, is a programming language used to form relationships with databases. There are various Relational Database Management System (RDBMS) that have work with SQL such as MySQL, Oracle, Microsoft SQL Server, PostgreSQL, Informix, MariaDB, MongoDB, and others.

MySQL is an excellent open source RDBMS product in web applications. Over time, MySQL has proven to exceed the performance of other RDBMS. MySQL also has features that are required by enterprise level applications. Although MySQL has a fairly large table size (8TB) per table, MySQL can operate on Intel Pentium class computers that have a minimum of 32MB RAM. MySQL underpins the SQL-92 standard almost totally and expands the standard with different highlights [5].

\subsection{ACID Transactions}

The ACID transaction is an idea that was first presented during the 1960s (in spite of the fact that the term ACID was not presented until 1983) [6]. In 1983 ACID was created by Andreas Reuter and Theo Harder which stand for Atomicity, Consistency, Isolation, and Durability [7]. An ACID transaction is a grouping of actions or operations that together have the following properties:

- Atomicity - Atomicity is the atomically action that implies indivisibility and irreducibility, so the transaction is guaranteed. Whereas the transaction is successful there will be a change and if the transaction fails then no change will occur and the transaction will never happened [8], [9].

- Consistency - Consistency implies when any given transaction start changes a consistent condition of the database or the data into another consistent condition till it ends, without essentially saving consistency at all intermediate points. This consistent state occur in spite the transaction succeeding or failing and both before the beginning the transaction and after the transaction is finished [10]-[12].

- Isolation - Transaction often executed simultaneously. Isolation guarantees that simultaneous execution of exchanges leaves the database in a similar condition that would have been acquired if the transactions were executed sequentially. It was done by concealed the transaction from every single other exchange until such time as the given exchange submits. So the transactions that run simultaneously have all the earmarks of being serialized [10][12]. 
- Durability - Durability means when the transaction has been carried out the changes will persist, even if there is a system failure. For example one account made money transactions to another account; this durability property guarantees that the update that already made won't be reversed [12].

\section{Method}

This research and development utilizes the waterfall method. Waterfall method is regularly called the exemplary life cycle (classic life cycle), where it shows a precise and consecutive way to deal with programming improvement, beginning with the specification of client needs and afterward proceeds through the phases of arranging (requirements), planning programs (design), convert the plan to code to make the program (implementation), then test the system (verification), which closures with help for the complete software that is delivered (maintenance). The phases of the waterfall method can be found in "Fig.1," beneath.

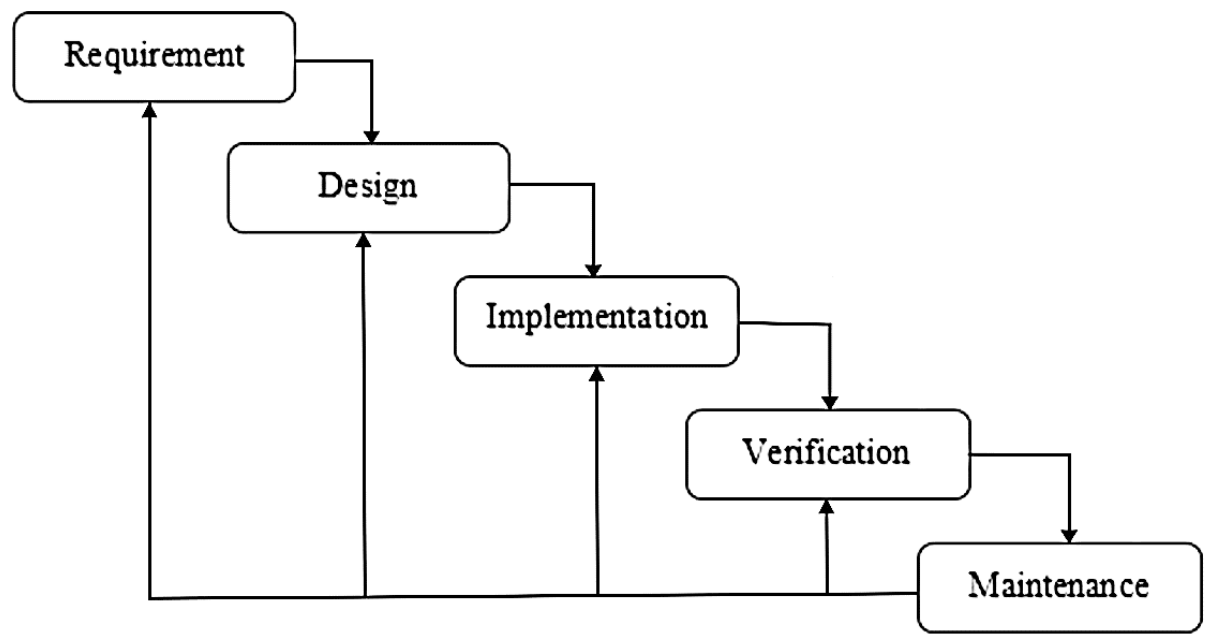

Fig. 1.Images of Waterfall Methodology

We chose this method because there are several advantages contained in this method other than because this method is an easy method to implement. The first advantage is that we can clearly get what is needed during the process of making this program before the creation begins. Because of the clear needs at the beginning of the process, each stage is completed within the allotted time. Referring to the level of difficulty of the implementation of this method which is quite low, then the level of resources needed is also quite low. But the problem arises if in the allotted time at the time of work each stage is not enough to complete the stage. Therefore it is necessary to prepare and calculate sufficiently mature to do this research using this waterfall method [13]. A complete description of the steps of the waterfall system is as follows:

\subsection{Requirement Analysis}

Requirement stage is a stage that analysis of system requirements to be made and must be understood by clients and developers. At this stage the client must be able to explain and define the purpose of the system to be built. As a developer must be able to capture the intent of the client regarding the system to be built and also provide advice and perhaps also constraints on the system to be built. The documents produced at this stage form the basis of a work contract between the client and the developer. This method is applied by researching and inputting data that will be made points in the questionnaire entries.

\subsection{System Design}

The prerequisites detail from the past stage will be examined in this stage and the system design is readied. System Design helps in deciding the (equipment) and system necessities and furthermore helps in characterizing the general system engineering. The system design stage is redefining by make and organizes the question points to form a questionnaire that is ready to be circulated. 


\subsection{Implementation}

Implementation is the stage where the general system design that has been arranged already will be changed over into program codes and modules which will later be incorporated into a complete system as per the work contract. At this stage programmers opens MySQL and direct the respondents to make an action with transactions and without transactions on MySQL.

\subsection{Verification}

At this stage the system that has been caused will to be incorporated and tried to test whether the system is working appropriately and that is no less significant is as per the agreement that has been concurred. The verification stage is carried out by respondents filling up the questionnaires

\subsection{Maintenance}

The final stage in the waterfall model. Finished software, run and maintain. Maintenance is incorporated into amending blunders that were not found in the past advance. Improvement of system unit execution and improvement of system services as new needs. At this stage the result of the questionnaires is being analyzed, charted and be concluded.

\section{Result and Discussion}

Before we go to the result, we gonna discuss how the transaction in MySQL works. A transaction is an atomic unit of database operations (Queries) in one or more databases that can be committed or rolled back. In Fig.2, it could be seen that when a transaction makes multiple queries to the database, either all the queries executed successfully when the transaction is committed, or any of the query failed when the transaction is rolled back.

A transaction begins with the initiate of transaction then the first executable query continues with other queries and ends when it is committed or rolled back. In a set of operations, if one of them fails, the rollback occurs to restore the database to its original state. If no error occurs, the entire set of statements is committed to the database.

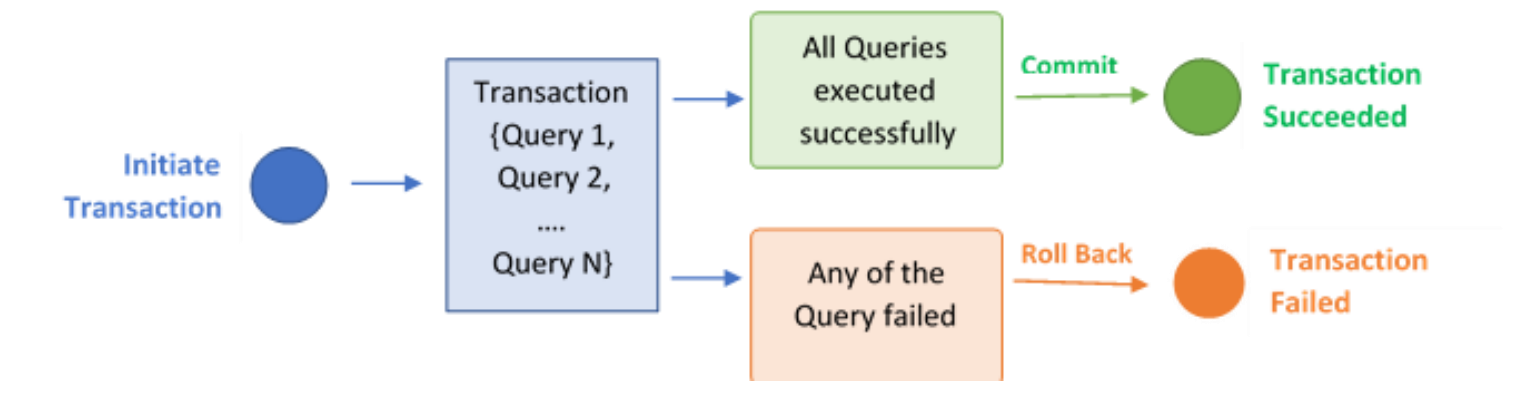

Fig. 2.Transaction Workflow

The advantages of using transaction, MySQL transaction allow you to execute a set of MySQL operations to ensure that the database never contains the result of partial operations. Imagine what would happen if in a transaction with multiple queries, one or more query failed due some reasons. It difficult to track and repair or make a return as the initial conditions where all steps should be successful because the data are related to each other. The only restoration option available is to reconstruct the data from a backup (providing one exists).

\subsection{Network Load Traffic Test Results on MySQL Atomic Transaction Database}

The test was carried out by means of 300 respondents accessing 2 different systems, one system using transactions and one other system did not use transactions. Researchers get the result that the densest network is a network owned by a system that uses transaction features, because there is an increase of about $13 \%$ of network traffic when compared to systems without transaction features. In Fig. 3, it could be seen that there is a difference in network load traffic between using transactions and not using transactions. 


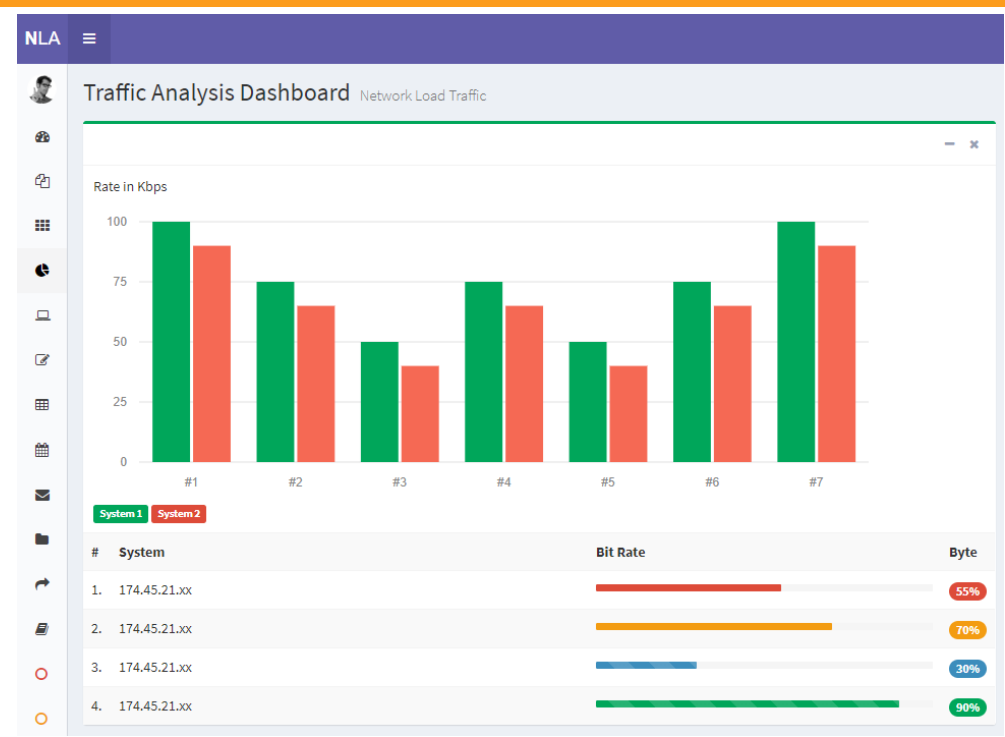

Fig. 3. Test Results

\section{Conclusions}

This research was conducted to determine network load when using transactions on MySQL. This research answers the purpose of algorithm design with transactions which has insignificant influence on network load but it is considered that if a large number of users access the system, congestion can occur. The results showed that there was a 13\% surge in traffic with 300 people in the trial, but this change in traffic was not linear with the number of users. Other researchers might be able to contribute by finding a prediction line from the surge in traffic.

\section{References}

[1] T. Benson, A. Akella, and D. A. Maltz, "Network traffic characteristics of data centers in the wild," in Proceedings of the 10th ACM SIGCOMM conference on Internet measurement, 2010, pp. 267-280.

[2] I. A. T. Hashem, I. Yaqoob, N. B. Anuar, S. Mokhtar, A. Gani, and S. U. Khan, "The rise of 'big data' on cloud computing: Review and open research issues," Inf. Syst., vol. 47, pp. 98-115, 2015.

[3] M. Troester, "Big Data Meets Big Data Analytics," 2012.

[4] Technopedia, "Network Traffic," www.technopedia.com, 2019. [Online]. Available: https://www.techopedia.com/definition/29917/network-traffic. [Accessed: 14-Sep-2019].

[5] S. Suehring, MySQL bible. John Wiley \& Sons, Inc., 2002.

[6] J. Gray and A. Reuter, Transaction processing: concepts and techniques. Elsevier, 1992.

[7] M. Chessell, C. Griffin, D. Vines, M. Butler, C. Ferreira, and P. Henderson, "Extending the concept of transaction compensation," IBM Syst. J., vol. 41, no. 4, pp. 743-758, 2002.

[8] J. Amsterdam, “Atomic File Transactions, Part 1," O'Reilly on java.com, 2001. [Online]. Available: https://web.archive.org/web/20160303090517/http://archive.oreilly.com/pub/a/onjava/2001/11/07/atomi c.html. [Accessed: 14-Sep-2019].

[9] S. Yu, "Acid properties in distributed databases," Adv. Ebus. Trans. B2B-Collaborations, p. 17, 2009.

[10] International Business Machines Corporation (IBM), "ACID properties of transactions," www.ibm.com, 2019. .

[11] Technet Microsoft, "Isolation Levels in the Database Engine," technet.microsoft.com, 2012. .

[12]C. J. Date, SQL and relational theory: how to write accurate SQL code " O'Reilly Media, Inc.," 2011.

[13]M. Ally, V. Balaji, A. Abdelbaki, and R. Cheng, "Use of Tablet Computers to Improve Access to Education in a Remote Location," J. Learn. Dev., vol. 4, no. 2, pp. 221-228, 2017. 